\title{
A COUPLED RANS-VOF AND FINITE ELEMENT MODEL FOR WAVE INTERACTION WITH HIGHLY FLEXIBLE VEGETATION
}

\author{
Haifei Chen¹, Qingping Zou* and Zhilong Liu ${ }^{1}$
}

\begin{abstract}
This paper presents a coupled wave-vegetation interaction model suitable for very flexible vegetation with large deflections. The wave hydrodynamics is modeled by a Navier-Stokes flow solver along with a Volume of Fluid surface capturing method. The governing equation for flexible vegetation motion is solved by a Finite Element Method using a semi-implicit time differencing scheme. The coupling between wave hydrodynamics and vegetation motion is achieved using an immersed boundary method. The model is validated with experimental measurements for a single-stem vegetation and a large-scale vegetation patch in a wave flume.
\end{abstract}

Keywords: wave interaction with vegetation; flexible vegetation; large deflection; IHFOAM; OpenFOAM; RANSVOF; finite element method; immersed boundary method

\section{INTRODUCTION}

Vegetation plays an important role in protecting natural shoreline against storm surge and waves. Majority of previous studies of wave-vegetation interaction focus on fixed vegetation. Li \& Yan (2007) and Marsooli \& Wu (2014) considered rigid vegetation in their Reynolds Averaged Navier-Stokes (RANS) models and neglected the swaying motion of the vegetation. Maza et al. (2013) used a twodimensional RANS model coupled with a submerged vegetation model, which solves only the displacement at the top of each stem and assumes a linear variation of deflection along the stem. Zhu and Chen (2015) coupled a non-hydrostatic phase resolving wave model, NHWAVE, with a Finite Element Method (FEM) based vegetation model. The complete force balance equation for the vegetation motion was solved. Their vegetation model is suitable for small deflections only.

The objective of this study is to develop a new coupled wave and vegetation model suitable for flexible vegetation with large sway of motion. A Reynolds Averaged Navier-Stokes solver with Volume of Fluid surface capturing scheme (RANS-VOF) is extended to include the effect of flexible vegetation on flow. The vegetation is assumed to behave like a slender rod, whose governing equations are solved by Finite Element Method (Garrett 1982; Chen et al. 2011). There is no constraint with regard to the magnitude of the vegetation motion in this coupled model as with most previous wave-vegetation models.

\section{NUMERICAL MODEL}

\section{FEM Model for Flexible Vegetation}

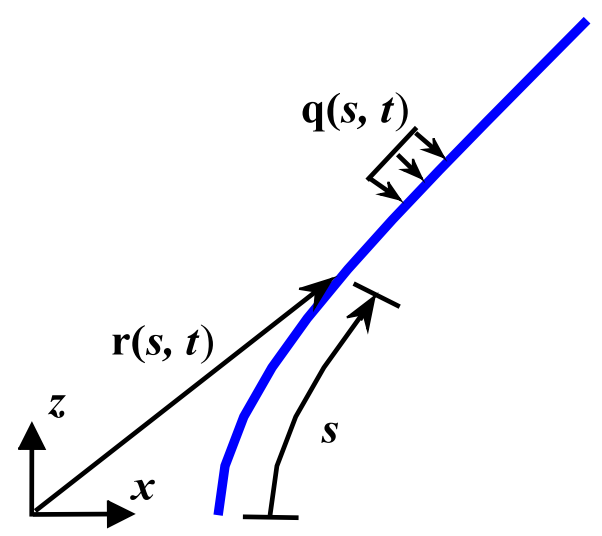

Figure 1. Coordinate system of the rod model for flexible vegetation. $r(s, t)$ is a position vector of the rod's centerline, $s$ is the arc length, and $q(s, t)$ is distributed force per unit length.

\footnotetext{
${ }^{1}$ Civil and Environmental Engineering, University of Maine, 5711 Boardman Hall, Orono, ME 04473, USA.

* qingping.zou@maine.edu
} 
The flexible vegetation is treated as a slender rod. The governing equation for the vegetation motion considers the buoyancy, stiffness, and gravity as restoring forces, drag and inertia as driving forces, and is given by

$$
-\left(E I \mathbf{r}^{\prime \prime}\right)^{\prime \prime}+\left(\lambda \mathbf{r}^{\prime}\right)^{\prime}+\mathbf{q}=m_{v} \ddot{\mathbf{r}}
$$

where $\mathbf{r}(s, t)$ is the position vector of the rod, the prime and dot denote the differentiation with respect to arc length $s$ and time $t$, respectively, $E I$ is bending stiffness, $m_{v}$ mass per unit length, $\lambda(s, t)=T_{e}-E I \kappa^{2}$ with $T_{e}$ being the local effective tension and $\kappa=\left|\mathbf{r}^{\prime \prime}\right|$ rod curvature. The external distributed load on the vegetation, $\mathbf{q}=\mathbf{w}+\mathbf{F}_{\mathbf{s}}+\mathbf{F}_{\mathbf{d}}$, is the sum of self-weight, $\mathbf{w}$, hydrostatic force, $\mathbf{F}_{\mathrm{s}}$, and hydrodynamic force, $\mathbf{F}_{d}$, acting on a unit length of single-stem vegetation. The hydrodynamic force on a single-stem vegetation is given by the Morrison equation,

$$
\mathbf{F}_{\mathrm{d}}=\frac{1}{2} \rho_{v} C_{D} b_{v}\left|\mathbf{U}^{\mathbf{n}}-\dot{\mathbf{r}}^{\mathbf{n}}\right|\left(\mathbf{U}^{\mathbf{n}}-\dot{\mathbf{r}}^{\mathrm{n}}\right)+b_{v} t_{v} \rho_{v} C_{M} \dot{\mathbf{U}}+\frac{1}{4} \pi b_{v}^{2} \rho_{v} C_{A}\left(\dot{\mathbf{U}}^{\mathbf{n}}-\ddot{\mathbf{r}}^{\mathbf{n}}\right)
$$

where $C_{D}, C_{M}$, and $C_{A}$ are the drag, inertia, and added mass coefficients, $\rho_{v}$ is the density of the vegetation, $b_{v}, t_{v}$ are the width and thickness of the rectangular cross-section of the vegetation blade,

$\mathbf{U}, \dot{\mathbf{U}}$ are fluid velocity and acceleration, $\dot{\mathbf{r}}, \ddot{\mathbf{r}}$ are the rod velocity and acceleration, the superscript " $n$ " indicates the normal component of the variable perpendicular to the rod.

FEM is used to discretize the governing equations. For static problems such as vegetation reconfiguration under current action, the Newton-Raphson method is used to solve the equations. For dynamic problems where transient wave motion is involved, a semi-implicit time differencing scheme is used. Readers should refer to Garret (1982) and Ran (2000) for more details.

\section{RANS-VOF Wave Model}

The Reynolds Averaged Navier-Stokes Solver coupled with a Volume of Fluid surface capturing scheme has been used extensively to solve free surface flow problems with large and complex deformations of free surface (Lin and Liu 1998; Lubin et al. 2006; Christensen 2006; Lv et al. 2009; Wang et al. 2009; Zou and Peng 2011). A Navier-Stokes solver built on the OpenFOAM platform is adopted to simulate the wave hydrodynamics. The open-source package, IHFOAM, can actively generate and absorb waves at the inlet and outlet boundaries (Higuera et al. 2013). The Volume of Fluid (VOF) method is used to capture the air-water interface. The governing equations for the RANS-VOF solver are given by

$$
\begin{aligned}
& \nabla \cdot \mathbf{U}=0 \\
& \frac{\partial \rho \mathbf{U}}{\partial t}+\nabla \cdot(\rho \mathbf{U U})-\nabla \cdot\left(\mu_{e f f} \nabla \mathbf{U}\right)=-\nabla p^{*}-\mathbf{g} \cdot \mathbf{X} \nabla \rho+\nabla \mathbf{U} \cdot \nabla \mu_{e f f}-\mathbf{F}_{\mathbf{d}} \\
& \frac{\partial \alpha}{\partial t}+\nabla \cdot(\mathbf{U} \alpha)+\nabla \cdot\left[\mathbf{U}_{r} \alpha(1-\alpha)\right]=0
\end{aligned}
$$

where $\rho$ is the fluid density, $p^{*}$ the pseudo-dynamic pressure, $\mathbf{g}$ the gravitational acceleration, $\mathbf{X}$ the position vector, $\mu_{e f f}=\mu+\rho v_{t}$ is the effective dynamic viscosity, which takes into account of molecular dynamic viscosity $\mu$ and the turbulent eddy viscosity $v_{t}$. Note that the vegetation-induced hydrodynamic forces, $\mathbf{F}_{\mathrm{d}}$, are added as a source term in the momentum equation (4). Eq. (5) is the advection equation for the volume of fluid function, $\alpha$, with an extra term to achieve a sharp interface (Weller 2002).

\section{Coupling Wave and Vegetation Models Using an Immersed Boundary Method}

The vegetation motion, Eq. (1), is solved by Finite Element Method considering drag and inertia as driving forces, while the RANS-VOF wave model considers the drag and inertia as a source term in the momentum equation (4). The two models are hence coupled through the vegetation-induced hydrodynamic forces. The common coupling approach used in the literature is based on the porous media flow theory, which considers the vegetated region as a porous media continuum and is thus concerned with only the volume-averaged flow field.

Different from previous studies using the porous media approach, we use an immersed boundary method suitable for a very thin object to account for the two way coupling of wave and vegetation (Perskin 2002). The force exerted by the vegetation on the flow, $-\mathbf{F}_{\mathbf{d}}$, is incorporated as a source term 
in the momentum equation (4), and is applied only wherever stands the vegetation stem. The immersed boundary method is a popular approach in simulating fluid-structure interactions (Lin 2007; Zhang et al. 2009).

Fig. 2 illustrates the flow chart of the procedure of solving the coupled wave-vegetation interaction model. At each time step of the computation, the vegetation motion is solved first with the known wave velocities. The vegetation deflection and displacement velocity are fed back to the wave model to calculate new flow velocities. Both the flow velocity and vegetation motion will then be fed to the vegetation model to obtain the new vegetation deflection and velocity at the next time step. The turbulence transport equations may be solved at the end of each time step.

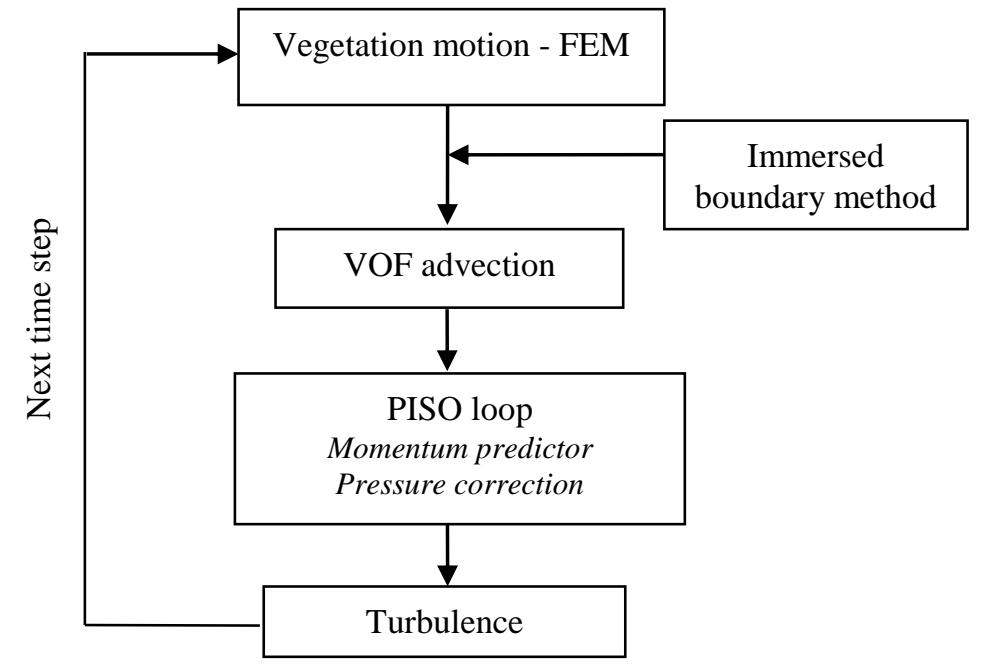

Figure 2. Flow chart of solving the coupled RANS-VOF flow model and FEM vegetation model.

\section{RESULTS AND DISCUSSION}

In this section, both the individual model components and the coupled wave-vegetation model is validated against the available experimental measurements. The IHFOAM wave model has been validated extensively by Higuera et al. (2013). The FEM model for flexible vegetation will be first validated using measurements for single-stem vegetation. The coupled model is then validated by a large-scale flume experiment of wave interaction with a vegetation patch.

\section{Wave Interaction with a Single-stem Flexible Vegetation}

Abdelrhman (2007) photographed Z. marina blades exposed to three different current speeds $U_{c}=$ $0.06,0.12$ and $0.14 \mathrm{~m} / \mathrm{s}$. Fig. 3 shows a comparison of the predicted and observed blade postures for this aquatic vegetation. The blade width and thickness are $b_{v}=0.8 \mathrm{~cm}$ and $t_{v}=0.35 \mathrm{~mm}$, blade length 40 $\mathrm{cm}$, and density $\rho_{v}=700 \mathrm{~kg} / \mathrm{m}^{3}$. The drag coefficient used is identical to that for flat plates, $C_{D}=1.95$. The predicted two blade postures are obtained using the higher $(E=2.4 \mathrm{GPa})$ and lower $(E=0.4 \mathrm{GPa})$ bound values of the elastic modulus for the blade. It is apparent that a higher elastic modulus corresponds to a more upright configuration. Given the uncertainty in the blade geometry and mechanical properties in the real vegetation, it can be stated that reasonable agreement is achieved between the predicted postures and the observations. 

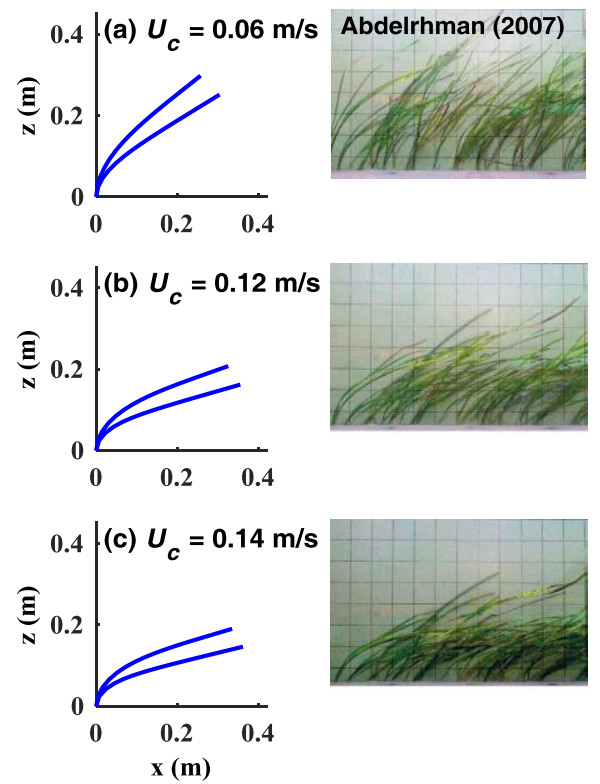

Figure 3. Comparison of model predictions for blade posture (left column) with the observations (right column) by Abdelrhman (2007) for the seagrass Zostera marina exposed to a current of speed $U_{c}$ (a) 0.06 $\mathrm{m} / \mathrm{s}$, (b) $0.12 \mathrm{~m} / \mathrm{s}$, and (c) $0.14 \mathrm{~m} / \mathrm{s}$.

Maza et al. (2013) conducted a set of experiments to measure the extent of plant motion under wave action. The vegetation mimics were composed of four PVC strips, one pair $45 \mathrm{~cm}$ long and another pair $27.5 \mathrm{~cm}$. The PVC strips were $1 \mathrm{~mm}$ thick and $1 \mathrm{~cm}$ wide, with a Young's modulus $E=0.9$ $\mathrm{GPa}$ and a density $\rho_{v}=700 \mathrm{~kg} / \mathrm{m}^{3}$. Since the two-pair, four-stem vegetation mimics respond freely to the wave action, it's difficult to determine the extent to which the four stems bind together to resist the wave action. To simplify the model simulation, an equivalent single-stem vegetation is used and model tests are thus used to determine the geometric and mechanical properties of the equivalent vegetation.

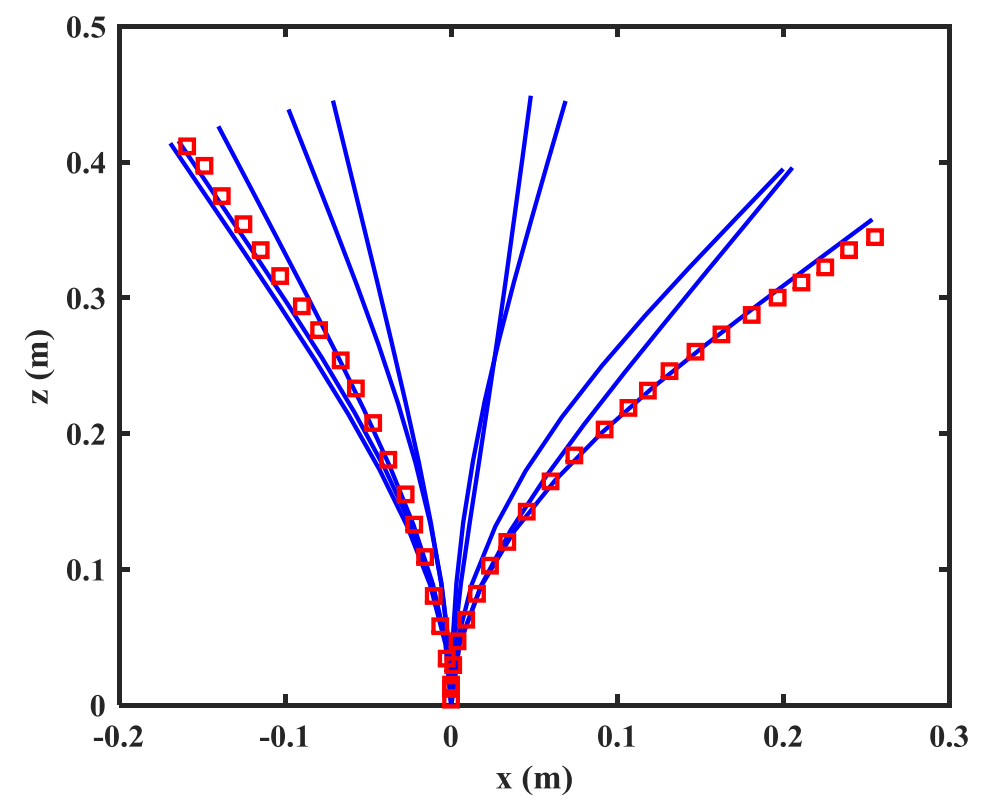

Figure 4. Instantaneous configuration of single-stem vegetation at every one tenth of the wave period under a periodic wave $T=4.0 \mathrm{~s}, H=0.2 \mathrm{~m}, d=0.8 \mathrm{~m}$. Red squares correspond to the measurements of the two extreme displacements under the wave motion by Maza et al. (2013).

Fig. 4 shows the predicted instantaneous configurations for the flexible vegetation over one wave period. The drag coefficient used is identical to that for flat plates, $C_{D}=1.95$. The bending stiffness 
used is equivalent to that when approximately $2 \mathrm{PVC}$ strips were binding together to resist the wave motion. The observed two maximum displacements of vegetation in the same and opposite direction as the wave direction are also shown as red squares in Fig. 4. The present model predictions are in good agreement with the measurements. Due to the asymmetry of wave kinematics, the vegetation flexibility and nonlinear wave-vegetation coupling, the vegetation motion is asymmetric relative to the vertical axis, i.e. the vegetation has a larger sway under wave crest $(\sim 0.25 \mathrm{~m})$ than under trough $(\sim 0.17 \mathrm{~m})$.

\section{Wave Interaction with a Patch of Flexible Vegetation}

The experimental measurements by Stratigaki et al. (2011) are used to validate the coupled wavevegetation model. Fig. 5 shows the present model setup simulating a regular wave, $\mathrm{T}=3.5 \mathrm{~s}$ and $\mathrm{H}=0.5$ $\mathrm{m}$, propagating through a vegetation meadow. The water depth is $2.4 \mathrm{~m}$ at the wavemaker boundary and transitions, through a $12 \mathrm{~m}$ long, $1: 17$ sandy slope, to $1.7 \mathrm{~m}$ at the onshore boundary. A $10.7 \mathrm{~m}$ long artificial vegetation meadow was placed over the flat portion of the sandy bottom. The beginning of this vegetation patch was located at $38.36 \mathrm{~m}$ from the wave paddle. The remaining sandy sloping beach beyond the vegetation patch is ignored in the simulation. The waves propagating through the vegetation field are absorbed by using proper outlet boundary conditions. The vegetation mimics used in this experiment were the same as described above (Maza et al. 2013). The vegetation density for this special case is $\mathrm{N}=180 \mathrm{stems} / \mathrm{m}^{2}$.

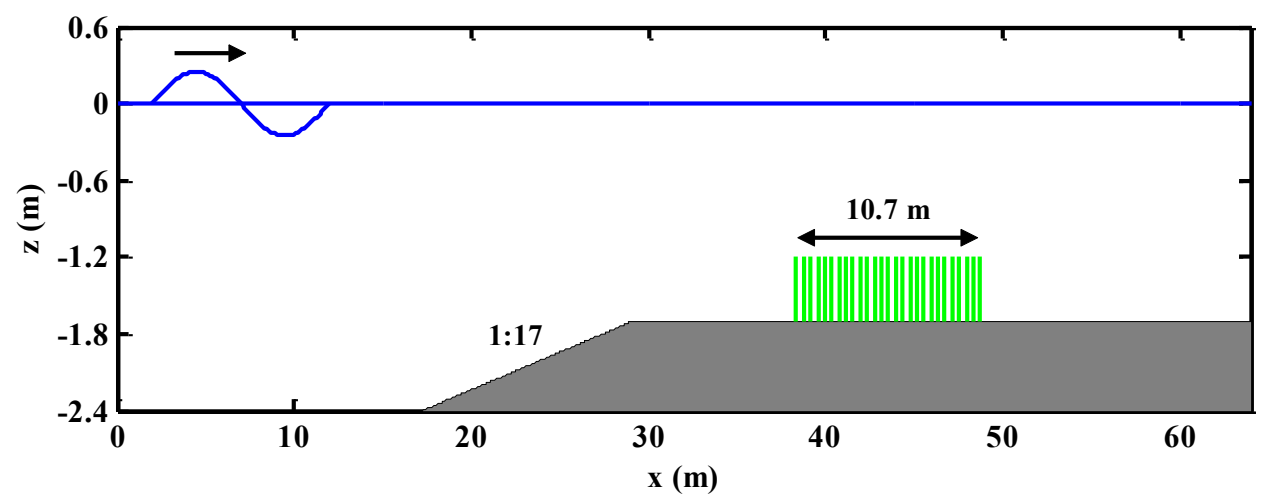

Figure 5. Model setup for coupled wave-vegetation interaction (Stratigaki et al. 2011).

Fig. 6 shows the spatial evolution of wave height over the vegetation patch. The $\mathrm{x}$-coordinate of the vegetation patch has been shifted to compare with experimental measurements easily. It is evident that the present model reproduces the trend of wave height decay as well as the magnitude of wave height oscillation over the vegetation field. However, the oscillation pattern is shifted probably due to the unknown wave reflection patterns in the physical flume not present in the numerical flume.

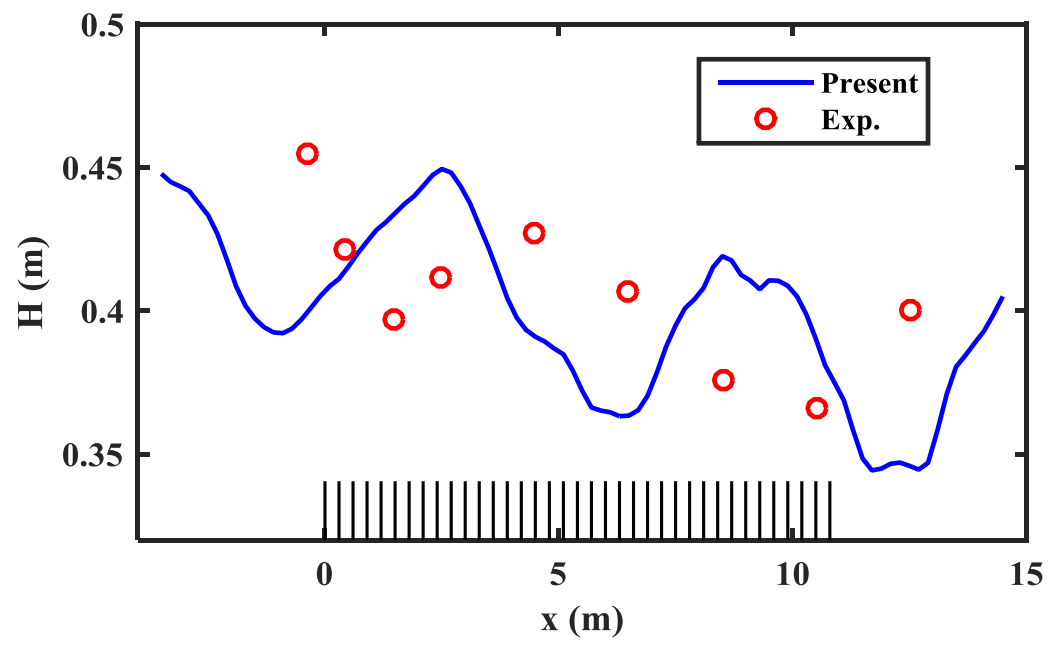

Figure 6. Predicted and measured (Stratigaki et al. 2011) spatial variation of wave height along the vegetation patch.

Fig. 7 presents the vertical profiles of horizontal and vertical wave velocity components at a location $2.0 \mathrm{~m}$ into the vegetation patch. It's seen that the vertical distribution of wave velocities are 
obviously altered by the presence of the vegetation, see Fig. 8 for illustration. Both the numerical predictions and the measurements show that the horizontal velocity increases around the upper end of the vegetation field. This skimming flow results from the strong discontinuity in the drag force between the area occupied by the meadow and the free flow above it. The strong discontinuity would cause a non-zero wave shear stress, leading to a mean current flowing in the direction of the wave propagation.
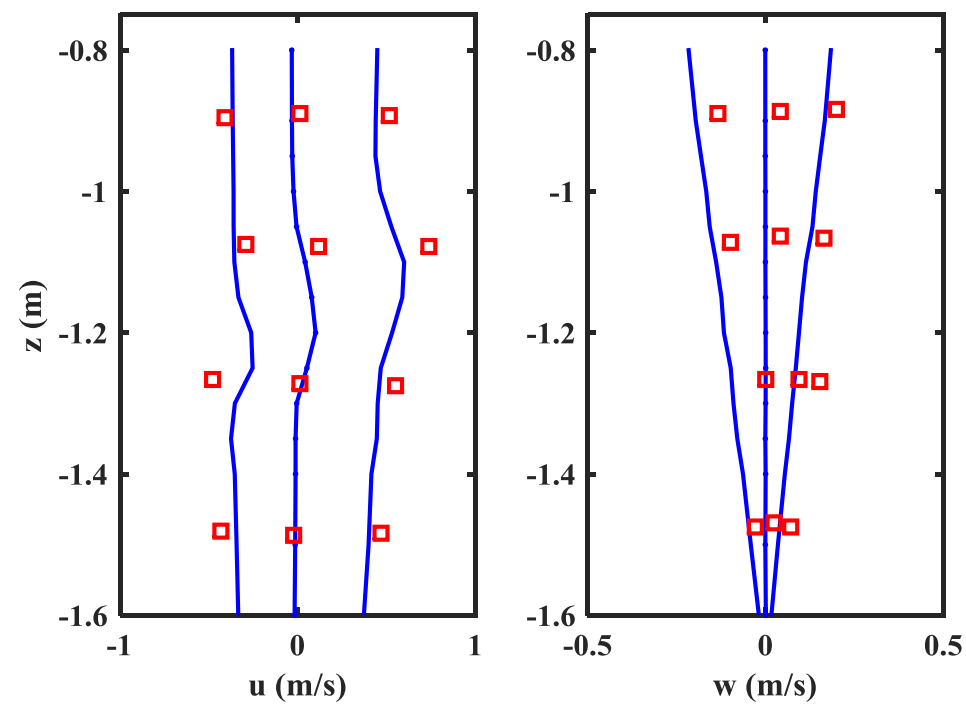

Figure 7. Model-data comparisons of vertical distribution of maximum, mean, and minimum (a) horizontal and (b) vertical wave velocities at a location $2.0 \mathrm{~m}$ into the vegetation patch. Solid lines: model prediction; squares: measurements by Stratigaki et al. (2011).

\section{Time: $53.0 \mathrm{~s}$}

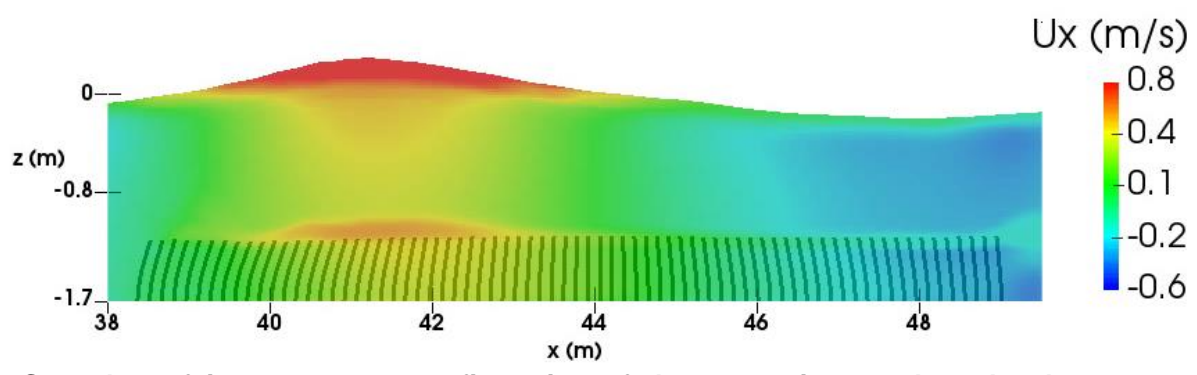

Figure 8. Snapshot of instantaneous configuration of the vegetation patch and color contour of wave horizontal velocity at $t=53 \mathrm{~s}$.

\section{CONCLUSIONS}

A coupled RANS-VOF and finite element model is developed to simulate the wave-vegetation interaction. The wave hydrodynamics is simulated by a RANS-VOF model. A Finite Element Method is adopted for solving the vegetation motion, which is applicable to flexible vegetation with either small or large deflections. The flexible vegetation model was validated with experiments for single-stem vegetation. The models of wave hydrodynamics and vegetation motion is coupled through a diffused immersed boundary method. The coupled model was validated with measurements for a large-scale flume experiment, where a regular wave propagating though a vegetation meadow was investigated. Both the observed wave height decay and oscillation magnitude and wave kinematics were reasonably predicted by the coupled model.

\section{REFERENCES}

Abdelrhman, M.A. 2007. Modeling coupling between eelgrass Zostera marina and water flow. Mar. Ecol. Prog. Ser. 338, 81-96.

Chen, H., S. Xu, and H. Guo. 2011. Nonlinear analysis of flexible and steel catenary risers with internal flow and seabed interaction effects. Journal of Marine Science and Application, 10(2), 156-162. 
Christensen, E.D. 2006. Large eddy simulation of spilling and plunging breakers. Coastal Engineering, $53(5-6), 463-485$.

Garrett, D.L. 1982. Dynamic analysis of slender rods, Journal of Energy Resources Tech., 104(4), 302306.

Higuera, P., J.L Lara, and I.J. Losada. 2013. Realistic wave generation and active wave absorption for Navier-Stokes models: application to OpenFOAM. Coast. Eng. 71, 102-118.

Li, C.W., and K. Yan. 2007. Numerical investigation of wave-current-vegetation interaction. $J$. Hydraul. Eng., (2007)133:7(794), 794-803.

Lin, P.Z., and P.L.-F. Liu. 1998. A numerical study of breaking waves in the surf zone. J. Fluid Mech., 359, 239-264.

Lin, P.Z. 2007. A fixed-grid model for simulation of a moving body in free surface flows. Computers \& fluids, 36(3), 549-561.

Lubin, P., S. Vincent, S. Abadie, and J.P. Caltagirone. 2006. Three-dimensional large eddy simulation of air entrainment under plunging breaking waves, Coastal Engineering, 53, 631-635.

Lv, X., Q.P. Zou, D.E. Reeve, and Y. Zhao. 2009. A novel coupled level set and volume of fluid method for sharp interface capturing on 3D tetrahedral grids, Journal of Computational Physics, 229(7), 2573-2604.

Marsooli, R., and W. Wu. 2014. Numerical investigation of wave attenuation by vegetation using a 3D RANS model. Advances in Water Resources, 74, 245-257.

Maza, M., J.L. Lara, and I.J. Losada. 2013. A coupled model of submerged vegetation under oscillatory flow using Navier-Stokes equations. Coast. Eng., 80, 16-34.

Peskin, C.S. 2002. The immersed boundary method. Acta numerica, 11, 479-517.

Ran, Z. 2000. Coupled dynamic analysis of floating structures in wave and current. Ph.D thesis, Texas A\&M University, College Station, TX.

Stratigaki, V., E. Manca, P. Prinos, I.J. Losada, J.L. Lara, M. Sclavo, C.L. Amos, I. Caceres, and A. Sanchez-Arcilla. 2011. Large-scale experiments on wave propagation over Posidonia oceanica. Journal of Hydraulic Research, 49, 31-43.

Wang, Z.Y., Q.P. Zou, and D.E. Reeve. 2009. Simulation of spilling breaking waves using a two phase flow CFD model, Computers \& Fluids, 38 (10), 1995-2005.

Weller, H.G. 2002. Derivation, modelling and solution of the conditionally averaged two-phase flow equations. Technical Report TR/HGW/02, OpenCFD Ltd.

Zhang, Y.L., Q.-P. Zou, D. Greaves, D. Reeve, A. Hunt-Raby, D. Graham, P. James, and X. Lv. 2010. A level set immersed boundary method for water entry and exit. Communications in Computational Physics, 8(2), 265-288.

Zhu, L., and Q. Chen. 2015. Numerical Modeling of Surface Waves over Submerged Flexible Vegetation. Journal of Engineering Mechanics, A4015001.

Zou, Q.-P., and Z. Peng. 2011. Evolution of wave shape over a low-crested structure, Coastal Engineering, 58(6), 478-488. 